\title{
Laryngeal squamous cell carcinoma at a South African referral hospital
}

\author{
Original \\ Article
}

\author{
M.E.Roos ${ }^{1}$, A. Sherriff ${ }^{2}$, M. Nel ${ }^{3}$, R.Y. Seedat ${ }^{1}$ \\ ${ }^{1}$ Department of Otorhinolaryngology, ${ }^{2}$ Department of Oncology, ${ }^{3}$ Department of Biostatistics, \\ University of the Free State, Bloemfontein, South Africa
}

\begin{abstract}
Introduction and aim: Laryngeal squamous cell carcinoma (LSCC) is the most common tumour of the head and neck region. The aim of this study was to determine the profile of patients with laryngeal carcinoma who presented to Universitas Academic Hospital over a 6 year period and to review their management.

Methods: This was a retrospective descriptive cross-sectional study. The records of 250 patients with LSCC between 1 January 2010 and 31 December 2015 were reviewed.

Results: The median age at presentation was 60.1 years, with $86.8 \%$ being males. Dysphonia was present in $99.6 \%$ of patients, but there was also a high prevalence of late symptoms. Most patients $(51.6 \%)$ presented with Stage IVa disease and $21.6 \%$ presented with Stage IVb disease. Nodal metastases were present in $60.4 \%$ of patients, while $4.8 \%$ had systemic metastases. There was high rate of patients who defaulted treatment and poor patient follow-up.

Conclusion: Late presentation is a significant problem at our institution with $95.6 \%$ of patients who presented with stage III and stage IV tumours.
\end{abstract}

Key Words: Laryngeal neoplasms, laryngectomy, radiotherapy, South Africa

Received: 9 January 2019, Accepted: 8 April 2019

Corresponding Author: Riaz Seedat, Department of Otorhinolaryngology, University of the Free State, Bloemfontein, South Africa Tel.: 27-51 405 3344, E-mail: seedatry@ufs.ac.za

ISSN: 2090-0740, July 2019 Vol.20, No.2

\section{INTRODUCTION}

Laryngeal squamous cell carcinoma (LSCC) is the most common tumour of the head and neck region. The clinical presentation of laryngeal carcinoma depends on the site and size of the tumour, with early glottic tumours presenting with hoarseness, while supraglottic tumours present with hoarseness, odynophagia or dysphagia ${ }^{[1]}$. Late stage tumours may present with airway obstruction $^{[2-6]}$. Treatment modalities that can be used for laryngeal carcinoma include surgery, radiotherapy, chemoradiotherapy and a combination thereof ${ }^{[1]}$. It has a good prognosis when detected early, and cure is possible with less aggressive treatment.

\section{AIM}

The aim of this study was to determine the profile of patients with laryngeal carcinoma who presented to Universitas Academic Hospital, a referral hospital in the Free State province of South Africa and review their management.

\section{METHODS}

This was a retrospective descriptive study of patients with laryngeal carcinoma who presented to the Department of Otorhinolaryngology at Universitas Academic Hospital between 1 January 2010 and 31 December 2015. The records of all patients were reviewed and demographic information, date of presentation, presenting symptoms, social habits, blood and histopathology results, staging, treatment and treatment outcomes were recorded on a dataform. Tumours were staged based on the patients' records according to the AJCC TNM Classification for Head and Neck Cancer 7th edition ${ }^{[7]}$.

Treatment failure was defined as residual disease within 12 weeks of completing treatment, while recurrence was defined as the presence of disease more than 12 weeks after completing treatment. Patients were considered to be lost to follow up when they did not follow up for the past 3 months during the first 2 years of completing treatment, for the past 6 months within 2 to 4 years of completing treatment and for the past year within 5 years of completing treatment. 
Descriptive statistics, namely frequencies and percentages for categorical data, and medians and percentiles for numerical data, were calculated. Associations were determined using Fisher's exact test and differences between groups were determined by means of the Kruskal-Wallis test.

The study was approved by the Health Sciences Research Ethics Committee of the University of the Free State (HSREC 38/2016). Permission to perform the study was obtained from the Free State Department of Health.

\section{RESULTS}

The median age at presentation was 60.1 years (range 30.5-83.7 years), with 217 (86.8\%) male and 33 (13.2\%) female patients.

Dysphonia was present in $99.6 \%$ of patients at presentation, but there was also a high prevalence of late symptoms, such as loss of weight, dyspnoea and dysphagia (Table 1). The duration of dysphonia was recorded in 169 patients, of whom $69(40.8 \%)$ had dysphonia for less than 6 months, $84(49.7 \%)$ had dysphonia for 6 to 12 months and $16(9.5 \%)$ had dysphonia for more than 12 months.

Table 1: Presenting symptoms $(\mathrm{n}=250)$

\begin{tabular}{ll}
\hline Symptom & Number of patients $(\%)$ \\
\hline Dysphonia & $249(99.6 \%)$ \\
Loss of weight & $106(42.4 \%)$ \\
Dyspnoea & $96(38.4 \%)$ \\
Dysphagia & $83(33.2 \%)$ \\
Stridor & $57(22.8 \%)$ \\
Odynophagia & $49(19.6 \%)$ \\
Cough & $36(14.4 \%)$ \\
Aspiration & $15(6 \%)$ \\
\hline
\end{tabular}

The smoking history was recorded in 246 patients, with $238(96.7 \%)$ having a history of smoking. Alcohol use history was recorded in 234 patients, of whom 197 $(84.2 \%)$ had a history of alcohol use. There was a history of both smoking and alcohol use in $195(83.3 \%)$ of the 234 patients for whom this information was documented while 6 patients $(2.6 \%)$ did not have a history of smoking or alcohol use.

Glottic tumours were present in 130 patients $(52.0 \%)$, supraglottic tumours in 113 patients (45.2\%) and subglottic tumours in 7 patients $(2.8 \%)$. The majority of patients presented with stage T4a (54.0\%) and T3 (24.4\%) tumours, while the most common lymph node staging was N0 (39.6\%) and N2c (24.4\%) (Table 2).
Table 2: $\mathrm{T}$ and $\mathrm{N}$ staging and combinations $(\mathrm{n}=250)$

\begin{tabular}{|c|c|c|c|c|c|c|}
\hline & & $\mathrm{T} 1$ & $\mathrm{~T} 2$ & T3 & $\mathrm{T} 4 \mathrm{a}$ & $\mathrm{T} 4 \mathrm{~b}$ \\
\hline & Total & $\begin{array}{c}5 \\
(2.0 \%)\end{array}$ & $\begin{array}{c}8 \\
(3.2 \%)\end{array}$ & $\begin{array}{c}61 \\
(24.4 \%)\end{array}$ & $\begin{array}{c}135 \\
(54.0 \%)\end{array}$ & $\begin{array}{c}41 \\
(16.4 \%)\end{array}$ \\
\hline N0 & $\begin{array}{c}99 \\
(39.6 \%)\end{array}$ & $\begin{array}{c}4 \\
(1.6 \%)\end{array}$ & $\begin{array}{c}5 \\
(2.0 \%)\end{array}$ & $\begin{array}{c}38 \\
(15.2 \%)\end{array}$ & $\begin{array}{c}41 \\
(16.4 \%)\end{array}$ & $\begin{array}{c}11 \\
(4.4 \%)\end{array}$ \\
\hline N1 & $\begin{array}{c}31 \\
(12.4 \%)\end{array}$ & $\begin{array}{c}1 \\
(0.4 \%)\end{array}$ & $\begin{array}{c}1 \\
(0.4 \%)\end{array}$ & $\begin{array}{c}5 \\
(2.0 \%)\end{array}$ & $\begin{array}{c}19 \\
(7.6 \%)\end{array}$ & $\begin{array}{c}5 \\
(2.0 \%)\end{array}$ \\
\hline N2a & $\begin{array}{c}18 \\
(7.2 \%)\end{array}$ & $0(0 \%)$ & $0(0 \%)$ & $\begin{array}{c}7 \\
(2.8 \%)\end{array}$ & $\begin{array}{c}10 \\
(4.0 \%)\end{array}$ & $\begin{array}{c}1 \\
(0.4 \%)\end{array}$ \\
\hline $\mathrm{N} 2 \mathrm{~b}$ & $\begin{array}{c}14 \\
(5.6 \%)\end{array}$ & $0(0 \%)$ & $0(0 \%)$ & $\begin{array}{c}1 \\
(0.4 \%)\end{array}$ & $\begin{array}{c}11 \\
(4.4 \%)\end{array}$ & $\begin{array}{c}2 \\
(0.8 \%)\end{array}$ \\
\hline $\mathrm{N} 2 \mathrm{c}$ & $\begin{array}{c}61 \\
(24.4 \%)\end{array}$ & $0(0 \%)$ & $\begin{array}{c}2 \\
(0.8 \%)\end{array}$ & $\begin{array}{c}7 \\
(2.8 \%)\end{array}$ & $\begin{array}{c}39 \\
(15.6 \%)\end{array}$ & $\begin{array}{c}13 \\
(5.2 \%)\end{array}$ \\
\hline N3 & $\begin{array}{c}27 \\
(10.8 \%)\end{array}$ & $0(0 \%)$ & $0(0 \%)$ & $\begin{array}{c}3 \\
(1.2 \%)\end{array}$ & $\begin{array}{c}15 \\
(6.0 \%)\end{array}$ & $\begin{array}{c}9 \\
(3.6 \%)\end{array}$ \\
\hline
\end{tabular}

Twelve patients (4.8\%) had systemic metastatic disease at presentation; 10 had pulmonary metastases, 1 had liver metastases and 1 had both liver and pulmonary metastases. All patients with metastases had T4 tumours. More than three-quarters $(78.0 \%)$ of patients presented with stage IV disease, with $51.6 \%$ having Stage IVa disease at presentation (Table 3). On histological examination, most patients $(70.4 \%)$ had moderately differentiated SCC (Table 4). One patient had a papillary SCC and HPV16 was identified by PCR from this tumour.

Table 3: Tumour stage $(\mathrm{n}=250)$

\begin{tabular}{ll}
\hline Stage & Number of patients $(\%)$ \\
\hline I & $5(2.0 \%)$ \\
II & $6(2.4 \%)$ \\
III & $44(17.6 \%)$ \\
IVa & $129(51.6 \%)$ \\
IVb & $54(21.6 \%)$ \\
IVc & $12(4.8 \%)$ \\
\hline
\end{tabular}

Table 4: Histological findings $(\mathrm{n}=250)$

\begin{tabular}{ll}
\hline Histology & Number of patients (\%) \\
\hline Well differentiated SCC & $42(16.8 \%)$ \\
Moderately differentiated SCC & $176(70.4 \%)$ \\
Poorly differentiated SCC & $22(8.8 \%)$ \\
SCC not otherwise specified & $6(2.4 \%)$ \\
SCC in situ & $3(1.2 \%)$ \\
Papillary SCC & $1(0.4 \%)$ \\
\hline
\end{tabular}

The median haemoglobin level was $12.9 \mathrm{~g} / \mathrm{dL}$ (range 6.3-18.1 g/dL) ( $\mathrm{n}=240)$, with 157 (65.4\%) patients being anaemic. The median albumin level was $32 \mathrm{~g} / \mathrm{L}$ (range 14 $46 \mathrm{~g} / \mathrm{L})(\mathrm{n}=215)$, with $144(67.0 \%)$ patients having albumin levels below normal. Twenty-eight patients (13.7\%) were 
Human Immunodeficiency Virus (HIV) positive, with a median CD4 count of 266 cells/mm3 (range 66-725 cells/ $\mathrm{mm} 3)$, while $176(86.3 \%)$ were HIV negative $(\mathrm{n}=204)$.

Tracheostomies were performed in 195 (78.0\%) patients and $36(14.4 \%)$ patients had feeding tubes inserted.

A total of 205 patients $(82.0 \%)$ received treatment, 40 patients $(16.0 \%)$ defaulted before initiation of treatment and five patients $(2.0 \%)$ demised before treatment could be initiated.

Surgery was performed in $53(21.2 \%)$ patients (Table 5); $46(86.8 \%)$ patients had total laryngectomies, $4(7.5 \%)$ patients had transoral laser surgery, $2(3.8 \%)$ patients had cold steel excision biopsies and 1 (1.9\%) patient had a partial laryngectomy at another hospital prior to referral.
Surgical complications in patients who had total laryngectomies included pharyngocutaneous fistulas in 6 patients $(13.3 \%)$, and one patient each $(2.2 \%)$ with wound sepsis, stomal stenosis and oesophageal stenosis. There was no association between the development of a pharyngocutaneous fistula and tumour site $(p=0.6840)$, tumour stage $(p=1.000)$, tumour grade $(p=0.5465)$, presence of preoperative tracheostomy $(\mathrm{p}=1.000)$, or HIV status $(\mathrm{p}=0.5025)$. There was no significant difference between patients that developed pharyngocutaneous fistulas and those that did not with regard to age $(\mathrm{p}=0.2023)$, haemoglobin level $(\mathrm{p}=0.6715)$, or albumin level $(\mathrm{p}=0.7692)$. Of the patients who developed pharyngocutaneous fistulas, five had positive resection margins, and one had a salvage laryngectomy after radiotherapy.

Total radiotherapy doses of 60-70 Gy were given to 120 $(78.9 \%)$ of the patients who received definite radiotherapy

Table 5: Treatment Modalities per T staging

\begin{tabular}{|c|c|c|c|c|c|}
\hline & $\mathrm{T} 1(\mathrm{n}=5)(\%)$ & $\mathrm{T} 2(\mathrm{n}=8)(\%)$ & $\mathrm{T} 3(\mathrm{n}=62)(\%)$ & $\mathrm{T} 4 \mathrm{a}(\mathrm{n}=135)(\%)$ & $\mathrm{T} 4 \mathrm{~b}(\mathrm{n}=40)(\%)$ \\
\hline Definitive chemoradiotherapy & $2(40 \%)$ & $5(62.5 \%)$ & $27(43.5 \%)$ & $41(30.4 \%)$ & $18(45.0 \%)$ \\
\hline Definitive radiotherapy & & & $11(17.7 \%)$ & $35(25.9 \%)$ & $13(32.5 \%)$ \\
\hline Cold steel excision & $2(40.0 \%)$ & & & & \\
\hline Transoral laser surgery & $1(20.0 \%)$ & & & & \\
\hline Transoral laser surgery + radiotherapy & & & $1(1.6 \%)$ & & \\
\hline Transoral laser surgery + chemoradiotherapy & & & $2(3.2 \%)$ & & \\
\hline Partial laryngectomy + chemoradiotherapy & & & & $1(0.7 \%)$ & \\
\hline Total laryngectomy & & & $5(8.1 \%)$ & $4(3.0 \%)$ & \\
\hline Total laryngectomy + radiotherapy & & & $2(3.2 \%)$ & $15(11.1 \%)$ & \\
\hline Total laryngectomy + chemoradiotherapy & & & $4(6.5 \%)$ & $16(11.9 \%)$ & \\
\hline Defaulted & & $3(37.5 \%)$ & $10(16.1 \%)$ & $23(17.0 \%)$ & $9(22.5 \%)$ \\
\hline
\end{tabular}

Twenty-three (43.4\%) of the 53 surgical patients had post-operative adjuvant chemoradiotherapy and 18 patients $(34.0 \%)$ post-operative adjuvant radiotherapy. Twelve $(22.6 \%)$ patients did not receive adjuvant treatment. Three patients had transoral excision for T1 tumours and adjuvant treatment was not indicated. The 3 patients with T3 tumours who had laser excision all received adjuvant continuous radiotherapy with a median dose of 70 Gy. Nine patients who had total laryngectomies did not receive adjuvant treatment; 4 did not require adjuvant treatment, 4 defaulted adjuvant treatment and one died of a pulmonary embolus before adjuvant treatment.

The patients who underwent a total laryngectomy $(\mathrm{n}=46)$ had a median albumin of $34 \mathrm{~g} / \mathrm{L}$ (range 18-40 g/L) and a median haemoglobin of $12.9 \mathrm{~g} / \mathrm{dL}$ (range 8.7-17.1 $\mathrm{g} / \mathrm{dL}$ ). Sixty Gy post-operative adjuvant radiotherapy was administered as continuous radiotherapy to 35 (76.1\%) patients and as a split course in $2(4.3 \%)$ patients. Twenty $(54.1 \%)$ of these patients received concurrent chemotherapy. or chemoradiotherapy, while $32(21.1 \%)$ received doses below $60 \mathrm{~Gy}$. These were patients who received palliative treatment (a single course of hypofractionated radiotherapy of $30 \mathrm{~Gy}$ ), patients who defaulted treatment and those who demised before completion of treatment.

Treatment failure was documented in 15 patients $(7.3 \%)$ of the 205 patients who received treatment. The staging of these patients was: 1 stage II, 2 stage III, 10 stage IVa and 2 stage IVb. Four patients with treatment failure had total laryngectomies, 5 received definitive chemoradiotherapy and 6 definitive radiotherapy.

Recurrence was reported in $20(9.8 \%)$ of the 205 patients who were treated. Thirteen $(6.5 \%)$ patients had local recurrence and 7 (3.5\%) had metastases. The initial staging for these patients was: 4 stage III, 14 stage IVa and 2 stage IVb. Ten of the 11 surgical patients with recurrence had stage IVa disease and 1 had stage III disease. Four had received adjuvant chemoradiotherapy, 5 adjuvant radiotherapy and 2 defaulted adjuvant therapy. 
Three patients (1.2\%) developed second primary tumours, 2 in the oropharynx and 1 in the lung.

Fifteen of the $38(39.5 \%)$ patients with residual disease, recurrent disease or second primaries, received salvage therapy. Two patients received salvage surgery, 9 patients received palliative chemotherapy, 2 patients received palliative radiotherapy and 2 patients received palliative chemoradiotherapy. Salvage surgery included a total laryngectomy in 1 patient and a lymph node excision in 1 patient who had previously had a neck dissection.

Deaths were reported in $34(13.6 \%)$ patients. Regular follow-up was documented in only $45(18.0 \%)$ of the remaining patients who were alive at last follow up while 171 patients $(68.4 \%)$ were lost to follow-up. Survival data could therefore not be extrapolated.

\section{DISCUSSION}

The patient profile was predominantly elderly males with a history of smoking and alcohol use. Most patients had advanced stage tumours at presentation, presenting with late symptoms viz. dyspnoea, stridor and dysphagia, and haemoglobin and albumin levels below normal. There was a high rate of patients defaulting treatment and poor follow-up.

The median age of patients in our study was 60.1 years, similar to a previous study in our institution with a mean age of 61.9 years $^{[3]}$. Laryngeal carcinoma is usually diagnosed between the ages of 55 and 64 years ${ }^{[8]}$. The male:female ratio of laryngeal carcinoma ranges between $7: 1$ and $25: 1^{[8,9]}$. In our study $86.8 \%$ of patients were male and $13.2 \%$ were female.

The major risk factors for the development of laryngeal carcinoma include smoking and alcohol use ${ }^{[10]}$. Most $(96.8 \%)$ of our patients had a smoking history and $84.2 \%$ a history of alcohol use.

The clinical presentation of larynx carcinoma is dependent on the tumour site and $\operatorname{size}^{[1]}$. The most common presenting symptom in this study was dysphonia $(99.6 \%)$ for a median duration of 6 months, similar to a 2007 study in our institution where $98 \%$ of patients presented with hoarseness for a mean duration of 5 months before referral ${ }^{[3]}$. Other symptoms included loss of weight, dyspnoea, dysphagia, cough and aspiration. These presenting symptoms are similar to those in other developing countries ${ }^{[1]}$. In a Zimbabwean study, 50\% of patients presented 6 months after the onset of their symptoms ${ }^{[11]}$. In contrast, presentation with airway compromise is rare in the United Kingdom and only seen in extreme cases ${ }^{[1]}$.
Tracheostomies were required in $78.0 \%$ of patients and $14.4 \%$ had feeding tubes inserted. In a Nigerian study, $95.2 \%$ of patients with laryngeal carcinoma were tracheostomized at presentation because of upper airway obstruction $^{[5]}$. Possible reasons for the late presentation of patients at our institution include transport problems, delays in referral, socio-economic factors and lack of patient insight, similar to the problems encountered in a study by Adoga and Ma'an in Nigeria ${ }^{[5]}$.

More than three-quarters of our patients $(78.0 \%)$ had stage IV disease at presentation, similar to other studies from sub-Saharan Africa ${ }^{[2-6]}$. Although a worse survival has been demonstrated with organ preservation therapy in patients with stage IV disease ${ }^{[12]}$, only $39(30.2 \%)$ of patients with stage IVa disease had surgery, mainly due to patient choice and poor general condition at presentation, making them suboptimal surgical candidates.

More than a third of patients $(36.0 \%)$ presented with T3 and T4 tumours without nodal or metastatic spread. This trend was also seen in a Zimbabwean study where lymph nodes were rarely involved despite advanced stage tumours ${ }^{[11]}$. Possible explanations for this include a favourable host-tumour response resulting in a less aggressive tumour, the low incidence of cervical lymph node metastases in glottic carcinoma and underdetection of cervical metastases in patients where the neck was not staged radiologically ${ }^{[13,14]}$.

The high rate of advanced stage tumours at presentation seen in our study differs from developed countries where patients present with earlier stage tumours. In the USA, $60 \%$ of patients present with stage III to IV disease, compared to $96 \%$ in our study ${ }^{[10]}$. Lymph node metastases occurred in $60 \%$ of patients, compared to a rate of $40 \%$ in developed countries ${ }^{[15]}$, probably due to a higher rate of advanced stage tumours at presentation. Distant metastases were present in $4.8 \%$ of patients at presentation, most commonly to the lungs.

One patient had a papillary SCC, a SCC variant that is known to be associated with a less aggressive nature and a better prognosis ${ }^{[15,16]}$. Papillary SCCs of the larynx are associated with smoking, alcohol use and HPV infection and tend to occur more commonly in $\operatorname{males}^{[15,16]}$. The patient in our study with papillary SCC was a male with a history of smoking and alcohol use, and HPV16 was detected in the tumour. No patients in our study had verrucous carcinoma, a variant of SCC reported to make up 3\% of $\operatorname{LSCC}^{[17]}$.

Most patients had both haemoglobin (65.4\%) and albumin $(67.0 \%)$ levels below normal, and $9.2 \%$ of patients had haemoglobin levels below $10 \mathrm{~g} / \mathrm{dl}$. In patients with head 
and neck SCC, anaemia has been shown to be an important prognostic factor for tumour control and survival[18]. A low pre-treatment serum albumin level is associated with an increased risk of wound infection as well as poorer disease free survival, cancer-specific survival and overall survival in patients with head and neck cancer ${ }^{[18,19]}$.

The HIV infection rate of $13.7 \%$ is similar to the $12.7 \%$ prevalence of HIV in the general South African population $^{[20]}$. This finding suggests that LSCC is not an HIV-associated disease, in contrast to previous studies that have reported that HIV positive patients have an increased risk to develop LSCC compared to the general population $^{[21]}$.

Sixteen percent of patients were scheduled to start treatment, but defaulted. Possible reasons for this may include challenges with the transport system, poor clinical condition of patients making traveling to hospital difficult, poor understanding of the importance of receiving treatment and unwillingness to be away from home for the duration of treatment.

Early stage (T1 and T2) glottic and supraglottic tumours are best treated by single modality laryngeal preservation techniques such as transoral laser microscopic surgery, definitive radiotherapy, or chemoradiotherapy, with similar survival and long-term functional outcomes ${ }^{[1,22]}$. Open conservation procedures such as supraglottic laryngectomy and supracricoid laryngectomy are options for a limited number of patients due to the higher cost and morbidity ${ }^{[10]}$. The majority of patients $(53.8 \%)$ with early stage tumours received definitive chemoradiotherapy, $15.4 \%$ had cold steel excision, $7.7 \%$ had transoral laser surgery and $23.1 \%$ defaulted treatment (Table 5).

Treatment options for advanced stage tumours (T3 and T4) include radiotherapy, chemoradiotherapy, surgery or a combination thereof $f^{[23]}$. No consensus has been reached on the treatment of T3 tumours ${ }^{[24]}$. The majority $(61.3 \%)$ of patients with T3 tumours in our study received non-surgical larynx preservation treatment by means of chemoradiotherapy or radiotherapy. Surgery was performed in $22.6 \%$ of patients with T3 tumours.

A total laryngectomy is recommended in patients with a dysfunctional larynx and with tumour invasion through the thyroid cartilage ${ }^{[1,10]}$. Although T4 tumours have worse organ preservation rates and survival rates with nonoperative therapy ${ }^{[1,12]}, 56.3 \%$ of patients with T4a tumours received nonsurgical larynx preservation treatment, while $26.7 \%$ underwent surgery. Luo et al found a better rate of disease free survival with total laryngectomy for advanced tumours than with larynx preserving treatment, but a similar overall survival[ ${ }^{[23]}$.

Six patients (13.0\%) developed pharyngocutaneous fistulas post-laryngectomy. Risk factors for the development of a pharyngocutaneous fistula include previous radiotherapy, supraglottic tumour location, advanced stage tumours, positive resection margins and a post-operative haemoglobin level of less than $12.5 \mathrm{~g} / \mathrm{dL}^{[25]}$, but we did not find these factors to be associated with the development of pharyngocutaneous fistulas.

Radiotherapy is either used definitively for organ preservation, or as adjuvant treatment post-operatively. Adjuvant radiotherapy is indicated postoperatively for perineural and lymphovascular invasion, close margins of less than $1 \mathrm{~mm}$, subglottic extension, T4 tumours and multiple positive cervical lymph nodes ${ }^{[10]}$. Chemotherapy is added to radiotherapy in the case of positive resection margins or extracapsular nodal extension ${ }^{[10]}$. Conventional radiotherapy dosages range between 60 and 70 Gy administered in 2 Gy fractions, which is also used at our institution $^{[22]}$.

The addition of chemotherapy suppresses distant metastases and improves disease-free survival[ ${ }^{[22]}$, but it is not curative as a single modality treatment in head and neck cancer ${ }^{[26]}$. The superiority of chemoradiotherapy over radiotherapy alone, due to the radiosensitising effect of chemotherapy has been established ${ }^{[22,26]}$, and is therefore preferred despite its association with toxicity ${ }^{[1]}$. The benefit of induction chemotherapy has not been proven, but induction chemotherapy may be used to treat large volume symptomatic tumours before initiation of concurrent chemoradiotherapy ${ }^{[1]}$. At our institution, chemotherapy was used as concurrent chemoradiotherapy for primary treatment.

Recurrences occur in $25-50 \%$ of patients with advanced laryngeal cancer ${ }^{[27,28]}$. A recurrence rate of $23 \%$ was found in a Norwegian study of 1616 patients with LSCC ${ }^{[28]}$. In our study, 15 patients $(7.3 \%)$ had treatment failure and 20 $(9.8 \%)$ developed recurrent disease. All but one of these patients had advanced stage tumours. The poor follow-up of our patients probably led to an underdetection of residual and recurrent disease. The median time from completion of treatment to time of recurrence was 208 days. As recurrences usually occur within 3 years of the initial treatment ${ }^{[28]}$, the follow-up time in our study after treatment was probably not sufficient to detect all recurrences. Three patients $(1.2 \%)$ developed second primary tumours. This is lower than the $2-6 \%$ reported in the literature ${ }^{[29]}$, probably because of underdetection due to poor follow-up.

Salvage laryngectomy is the treatment of choice after failure of laryngeal preservation therapy ${ }^{[10]}$. Only one patient had a salvage laryngectomy, although more patients could have benefitted from this treatment. Possible reasons for this include poor patient condition, and unwillingness of patients to undergo surgery.

The majority of patients were lost to follow-up, therefore survival data could not be extrapolated. Possible 
reasons for not following up include deaths, transport problems and patients not being aware of the importance of follow-up.

Limitations of this study include the retrospective nature, limited sample size and large number of patients who were lost to follow-up.

\section{CONCLUSION}

The majority of patients presented with advanced stage tumours at presentation. There was a high rate of patients defaulting treatment and poor follow-up. There is a need for an improvement in health care services to establish earlier referral and patient education to improve patient compliance and follow-up and a need for further studies to look at reasons for late referral and poor follow-up of these patients.

\section{ACKNOWLEDGEMENTS}

We would like to thank Mr. M. Mamba for assistance with the statistical analysis.

\section{FINANCIAL SUPPORT}

This research did not receive any specific grant from funding agencies in the public, commercial, or not-forprofit sectors

\section{CONFLICT OF INTEREST}

The authors declare no conflict of interest

\section{REFERENCES}

1. Jones TM, De M, Foran B, Harrington $\mathrm{K}$, Mortimore S. Laryngeal cancer: United Kingdom National Multidisciplinary guidelines. J Laryngol Otol 2016;130(S2):S75-82.

2. Kitcher ED, Yarney J, Gyasi RK, Cheyuo C. Laryngeal cancer at the Korle Bu Teaching Hospital Accra Ghana. Ghana Med J 2006;40(2):45-9.

3. de Villiers G du T, Seedat RY, Claassen AJ, Joubert G. Laryngeal carcinoma - patient profile and delay in referral. S Afr Med J 2007;97(12):1274-5.

4. Somefun OA, Nwawolo CC, Okeowo PA, Alabi SB, Abdul-Kareem FB, Banjo AA, et al. Prognostic factors in the management outcome of carcinoma of the larynx in Lagos. Niger Postgrad Med J 2003;10(2):103-6.

5. Adoga A, Ma'an E. The challenges of treating laryngeal carcinoma in jos, Nigeria. J West African Coll Surg 2011;1(2):63-75.
6. Amusa YB, Badmus A, Olabanji JK, Oyebamiji EO. Laryngeal carcinoma: experience in Ile-Ife, Nigeria. Niger J Clin Pract 2011;14(1):74-8.

7. American Joint Committee on Cancer. AJCC Cancer Staging Manual. Edge SB, Byrd DR, Compton CC, Fritz AG, Green FL, Trotti A, editors. New York: Springer-Verlag; 2010.

8. Bradley PJ. Laryngeal cancer in nondrinker nonsmoker young patients: a distinct pathological entity? Curr Opin Otolaryngol Head Neck Surg 2016;24(2):140-7.

9. Menach P, Oburra HO, Patel A. Cigarette smoking and alcohol ingestion as risk factors for laryngeal squamous cell carcinoma at Kenyatta National Hospital, Kenya. Clin Med Insights Ear Nose Throat 2012;5:17-24.

10. Steuer CE, El-Deiry M, Parks JR, Higgins KA Saba NF. An update on larynx cancer. CA Cancer J Clin 2017;67(1):31-50.

11. Tumushime-Buturo CG, Bandason C, Makura ZG, Yousef OE. Cancer of the larynx at Harare Central Hospital in Zimbabwe. Cent Afr J Med 1993;39(8):167-70.

12. Britt CJ, Gourin CG. Contemporary management of advanced laryngeal cancer. Laryngoscope Investig Otolaryngol 2017;2(5):307-9.

13. Khoueir N, Matar N, Farah C, Francis E, Tabchy B, Haddad A. Survival of T4aN0 and T3N+ laryngeal cancer patients: a retrospective institutional study and systematic review. Am J Otolaryngol 2015;36(6):755-62.

14. Smee R, Williams JR, Kotevski DP. Management of locally advanced T3-4glottic laryngeal carcinomas. J Laryngol Otol 2018;132(7): 642-50.

15. Dutta R, Husain Q, Kam D, Dubal PM, Baredes S, Eloy JA. Laryngeal papillary squamous cell carcinoma: A population-based analysis of incidence and survival. Otolaryngol Head Neck Surg 2015;153(1):54-9.

16. Colby C, Klein AM. Papillary squamous cell carcinoma of the larynx. Ear Nose Throat J 2011;90(8):E13-5.

17. Thompson LDR. Laryngeal dysplasia, squamous cell carcinoma, and variants. Surg Pathol Clin 2017;10(1):15-33. 
18. Lim WS, Roh J-L, Kim S-B, Choi S-H, Nam SY, Kim SY. Pretreatment albumin level predicts survival in head and neck squamous cell carcinoma. Laryngoscope 2017;127(12):E437-42.

19. Danan D, Shonka DC, Selman Y, Chow Z, Smolkin ME, Jameson MJ. Prognostic value of albumin in patients with head and neck cancer. Laryngoscope 2016;126(7):1567-71.

20. Statistics South Africa. Statistical release P0302. Mid-year population estimates 2017. Pretoria; 2017.

21. McLemore MS, Haigentz M, Smith R V, Nuovo GJ, Alos L, Cardesa A, et al. Head and neck squamous cell carcinomas in HIV-positive patients: a preliminary investigation of viral associations. Head Neck Pathol 2010;4(2):97-105.

22. Hristov B, Bajaj GK. Radiotherapeutic management of laryngeal carcinoma. Otolaryngol Clin North Am 2008;41(4):715-40.

23. Luo X-N, Chen L-S, Zhang S-Y, Lu Z-M, Huang Y. Effectiveness of chemotherapy and radiotherapy for laryngeal preservation in advanced laryngeal cancer: a meta-analysis and systematic review. Radiol Med 2015;120(12):1153-69.
24. Riga M, Chelis L, Danielides V, Vogiatzaki T, Pantazis TL, Pantazis D. Systematic review on T3 laryngeal squamous cell carcinoma; still far from a consensus on the optimal organ preserving treatment. Eur J Surg Oncol 2017;43(1):20-31.

25. Liang JW, Li ZD, Li SC, Fang FQ, Zhao YJ, Li YG. Pharyngocutaneous fistula after total laryngectomy: A systematic review and metaanalysis of risk factors. Auris Nasus Larynx 2015;42(5):353-9.

26. Kelly CG. Chemotherapy: United Kingdom National Multidisciplinary Guidelines. J Laryngol Otol 2016;130(S2):S71-4.

27. Jin T, Lin H, Cai X, Wang H, Hu W, Guo L, et al. Treatment and prognosis of patients with recurrent laryngeal carcinoma: a retrospective study. Head Neck Oncol 2013;1-7.

28. Brandstorp-Boesen J, Sørum Falk R, Folkvard Evensen J, Boysen M, Brøndbo K. Risk of Recurrence in Laryngeal Cancer. PLoS One 2016;11(10):e0164068.

29. Hartl DM. Evidence-based practice: management of glottic cancer. Otolaryngol Clin North Am 2012;45(5):1143-61. 